\title{
Hochdosierte Brachytherapien beim Prostatakarzinom im Vergleich
}

\section{Bei Patienten mit Prostatakarzinom und günstigem Risikoprofil wurden die Nebenwirkungen von 3 unter- schiedlichen Regimes einer Hoch- dosis-Brachytherapie untersucht.}

nsgesamt 494 Patienten mit Prostatakarzinom des Stadiums $\leq \mathrm{T} 2 \mathrm{~b}$, einem Gleason-Score $\leq 7$ und einem PSA-Wert $\leq 15 \mathrm{ng} / \mathrm{ml}$ unterzogen sich einer alleinigen Hochdosis-Brachytherapie. 319 Patienten erhielten $38 \mathrm{~Gy}$ in 4 Fraktionen, 79 Patienten 24 Gy in 2 Fraktionen und 96 Betroffene 27 Gy in 2 Fraktionen. Die mediane Beobachtungsdauer lag bei 4 Jahren. Akute und chronische urogenitalen und gastrointestinalen Toxizitäten vom Grad $\geq 2$ ( $\leq 6$ bzw. $>6$ Monate nach Bestrahlung) waren in allen Gruppen vergleichbar.

Die häufigsten akuten und chronischen urogenitalen Toxizitäten waren häufiges Wasserlassen und Harndrang (Grad 1: $40 \%$ akut bzw. $37 \%$ chronisch,
Grad 2: $14 \%$ bzw. 30\%). Eine Dysurie trat bei $23 \%$ bzw. 19\% (Grad 1) und 6\% bzw. $7 \%$ (Grad 2) auf und Harnverhalt bei $16 \%$ bzw. $23 \%$ (Grad 1) und $7 \%$ bzw. $4 \%$ (Grad 2). Toxizitäten vom Grad 3 kamen nur selten vor, und es gab keine Toxizitäten der Grade 4 und 5.

Die häufigsten akuten und chronischen gastrointestinalen Toxizitäten waren $\mathrm{Di}$ arrhö (Grad 1: 9 bzw. 8\%). Andere akute gastrointestinale Toxizitäten vom Grad 1 traten bei weniger als $4 \%$ der Patienten auf, vom Grad 2 bei weniger als $1 \%$. Chronische rektale Blutungen vom Grad 1 wurden bei $7 \%$ der Patienten beobachtet, chronische rektale Schmerzen bei $5 \%$. Andere chronische gastrointestinale Toxizitäten der Grade 1 und 2 traten bei $\leq 2 \%$ der Patienten auf. Es wurden keine Toxizitäten der Grade 3-5 beobachtet. Hinsichtlich der Raten urogenitaler und gastrointestinaler Toxizitäten machte es keinen signifikanten Unterschied, ob die Patienten eine Androgendeprivation er- hielten oder nicht. Hinsichtlich der klinischen Ergebnisse (z. B. Überleben ohne biochemisches Versagen, krankheitsfreies Überleben, Gesamtüberleben) nach 5 Jahren zeigten sich keine Unterschiede zwischen den Brachytherapie-Schemata.

Fazit: Die 3 Hochdosis-BrachytherapieSchemata führten zu ähnlichen akuten und chronischen Toxizitäten, die insgesamt gut toleriert wurden. Es gab nur wenige Toxizitäten vom Grad 3 und keine vom Grad 4 oder 5. Dies, zusammen mit den vergleichbaren klinischen Ergebnissen, lässt schlussfolgern, dass alle drei Dosierungsschemata akzeptable Optionen für Patienten mit Prostatakarzinom und günstigem Risikoprofil sind.

Judith Neumaier

Jawad MS et al. Outcomes Associated With 3 Treatment Schedules of High-Dose-Rate Brachytherapy Monotherapy for Favorable-Risk Prostate Cancer. Int J Radiat Oncol Biol Phys. 2016(94)4:657-66.

\section{Prostatakarzinom: Dosisintensivierte Salvage- Radiotherapie nach biochemischem Versagen}

\author{
In einer Phase-III-Studie wurde untersucht, ob Patienten mit biochemischem \\ Versagen nach einer radikalen Prostatektomie von einer dosisintensivierten \\ Salvage-Radiotherapie des Prostatabetts profitieren.
}

B ei bis zu $40 \%$ der Patienten kommt es nach einer radikalen Prostatektomie binnen 5 Jahren zum biochemischen Versagen. Potenziell kurativ ist dann einzig die Salvage-Radiotherapie. Hier könnte eine Dosisintensivierung sinnvoll sein; inwiefern sie gastrointestinale (GI) und urogenitale Toxizitäten bedingt und die Lebensqualität beeinflusst, wurde nun untersucht.

Die 344 Patienten (48-75 Jahre) hatten ein biochemisches Therapieversagen nach radikaler Prostatektomie, aber keine Anzeichen einer makroskopischen Erkrankung. Sie erhielten randomisiert 64 oder 70 Gy - mit dreidimensionaler konformaler oder intensitätsmodulierter Bestrahlungs-/Rotationstechnik. Pri- märer Endpunkt war die Freiheit von biochemischem Versagen. 8,7\% der Patienten hatten zu Studienbeginn urogenitale Symptome vom Grad 2, 0,6 \% vom Grad 3, meist Harninkontinenz und Harndrang. Die Inkontinenz blieb $3 \mathrm{Mo-}$ nate nach Bestrahlung bei 56 Patienten unverändert, bei 7 verschlechterte und bei 46 verbesserte sie sich. Akute urogenitale Toxizitäten vom Grad $\geq 2$ wurden bei $13,6 \%$ der Patienten unter der 64-GyDosis beobachtet, bei $18,3 \%$ nach 70 Gy $(\mathrm{p}=0,2)$. Von den Patienten, die zu Studienbeginn noch potent waren, blieben 3 Monate nach der Bestrahlung 24,3\% (64 Gy) bzw. 30,8\% (70 Gy) potent.

GI-Toxizitäten vom Grad 2 hatten zu Studienbeginn 0,3\% der Patienten. Aku- te GI-Toxizitäten vom Grad 2 und 3 traten bei $16,6 \%$ der Patienten nach $64 \mathrm{~Gy}$ sowie bei $17,7 \%$ nach 70 Gy auf $(\mathrm{p}=0,8)$. In beiden Armen gaben vergleichbar viele Patienten nach 3 Monaten eine Verschlechterung bzw. Verbesserung der Lebensqualität an, wobei diese meist nur geringfügig und klinisch nicht relevant war. Patienten, die 70 Gy erhielten, gaben eine geringfügige klinisch relevante Verschlechterung von Harnwegssymptomen an, wohingegen diese Symptome unter 64 Gy stabil blieben.

Fazit: Eine Intensivierung der SalvageRadiotherapie war mit niedrigen Raten akuter urogenitaler und GI-Toxizitäten assoziiert. Der Einfluss auf die Lebensqualität blieb gering, klinisch relevant verschlechterten sich nur Harnwegssymptome leicht.

Judith Neumaier

Ghadjar P et al. Acute Toxicity and Quality of Life After Dose-Intensified Salvage Radiation Therapy for Biochemically Recurrent Prostate Cancer After Prostatectomy: First Results of the Randomized Trial SAKK 09/10. J Clin Oncol. 2015;33(35):4158-66. 